\title{
Localized Acceleration of Energetic Particles by a Weak Shock in the Solar Corona
}

\author{
David M. Long ${ }^{1}$ (D), Hamish A. S. Reid ${ }^{1}$ (D), Gherardo Valori ${ }^{2}$ (D), and Jennifer O'Kane ${ }^{1}$ (D) \\ ${ }^{1}$ Mullard Space Science Laboratory, UCL, Holmbury St. Mary, Dorking, Surrey, RH5 6NT, UK; david.long@ucl.ac.uk \\ ${ }^{2}$ Max Planck Institute for Solar System Research, Justus-von-Liebig-Weg 3, D-37077 Göttingen, Germany \\ Received 2021 May 5; revised 2021 August 10; accepted 2021 August 10; published 2021 November 1
}

\begin{abstract}
Globally propagating shocks in the solar corona have long been studied to quantify their involvement in the acceleration of energetic particles. However, this work has tended to focus on large events associated with strong solar flares and fast coronal mass ejections (CMEs), where the waves are sufficiently fast to easily accelerate particles to high energies. Here we present observations of particle acceleration associated with a global wave event which occurred on 2011 October 1. Using differential emission measure analysis, the global shock wave was found to be incredibly weak, with an Alfvén Mach number of $\sim 1.008-1.013$. Despite this, spatially resolved type III radio emission was observed by the Nançay RadioHeliograph at distinct locations near the shock front, suggesting localized acceleration of energetic electrons. Further investigation using magnetic field extrapolation identified a fan structure beneath a magnetic null located above the source active region, with the erupting CME contained within this topological feature. We propose that a reconfiguration of the coronal magnetic field driven by the erupting CME enabled the weak shock to accelerate particles along field lines initially contained within the fan and subsequently opening into the heliosphere, producing the observed type III emission. These results suggest that even weak global shocks in the solar corona can accelerate energetic particles via reconfiguration of the surrounding magnetic field.
\end{abstract}

Unified Astronomy Thesaurus concepts: Solar physics (1476); Solar corona (1483); Solar coronal waves (1995); Solar coronal radio emission (1993)

\section{Introduction}

Solar eruptions are the most energetic phenomena occurring in our solar system, emitting bursts of multi-spectral radiation (solar flares), ejecting massive bubbles of plasma into the heliosphere as coronal mass ejections (CMEs) and driving global shock waves that propagate through and restructure the surrounding corona (see Warmuth 2015; Long et al. 2017a). Although initially poorly understood, the advent of synoptic high-cadence, multi-wavelength Extreme Ultra-Violet (EUV) observations of the low solar corona from the Solar Dynamics Observatory (SDO; Pesnell et al. 2012) spacecraft has provided new insight into global waves in the solar corona. These global waves (formerly known as "EIT waves") are identified as bright annuli propagating away from the source of the eruption at typical velocities of $600-730 \mathrm{~km} \mathrm{~s}^{-1}$ (Nitta et al. 2013; Long et al. 2017a). While initially characterized using either wave or pseudo-wave interpretations (see Warmuth 2015), recent work (see Long et al. 2017b; Downs et al. 2021) has made it increasingly evident that these features can best be interpreted as large-amplitude waves/shocks. The multi-wavelength observations provided by SDO have also enabled plasma diagnostics of these perturbations, showing an adiabatic increase in both temperature and density associated with their passage through the solar corona (Vanninathan et al. 2015; Long et al. 2019; Frassati et al. 2020). However, in each case, these results have underlined the very weak nature of these shocks, finding Mach numbers only slightly greater than 1 (e.g., Long et al. 2015; Frassati et al. 2020).

The very low measured Mach numbers of these global shocks and the density of the corona through which they propagate suggests that these global shock waves should be subcritical and hence highly inefficient at accelerating particles. Despite this, previous work has related energetic particles detected in situ to global waves passing through the predicted footpoint of the connected magnetic field (see Rouillard et al. 2012; Prise et al. 2014). However, these connected observations are relatively rare due to mixing of energetic particles between their origin at the Sun and detection in situ near the Earth (see Laitinen \& Dalla 2019).

In addition to the direct detection of energetic particles accelerated by global waves, the advent of synoptic high-cadence, high-resolution observations of the Sun across the electromagnetic spectrum has also provided a unique opportunity to both identify and probe global waves and their relationship to other solar phenomena such as energetic particles. Both Carley et al. (2013) and Morosan et al. (2019) identified type III herringbone radio emission as corresponding to rippling in the laterally propagating front of a CME-driven shock wave (observed in the low corona as a global EUV wave), theorizing that this should enable significant particle acceleration by these weak global shocks. However, these signatures were associated with large solar eruptions, typically characterized by X-class flares, very fast CMEs $\left(v_{\mathrm{CME}} \sim 2000 \mathrm{~km} \mathrm{~s}^{-1}\right)$, and fast, strong global shock wave events. In contrast, most observed global shock wave events are much weaker, suggesting that these very high energy events may not provide the best insight into the processes by which energetic particles are typically accelerated by low-energy global shocks in the low corona. More recent work by Morosan et al. (2020) has shown evidence of extended radio emission produced by a solar eruption with an associated global wave front weaker than the events studied by Carley et al. (2013) and Morosan et al. (2019). However, this event erupted from the backside of the Sun and could only be studied from Earth once it appeared above the solar limb, thus complicating a full analysis of the global wave front.

Here we use a combination of multi-spectral observations and modeling to investigate the evolution of a weak global shock wave in the solar corona associated with spatially 

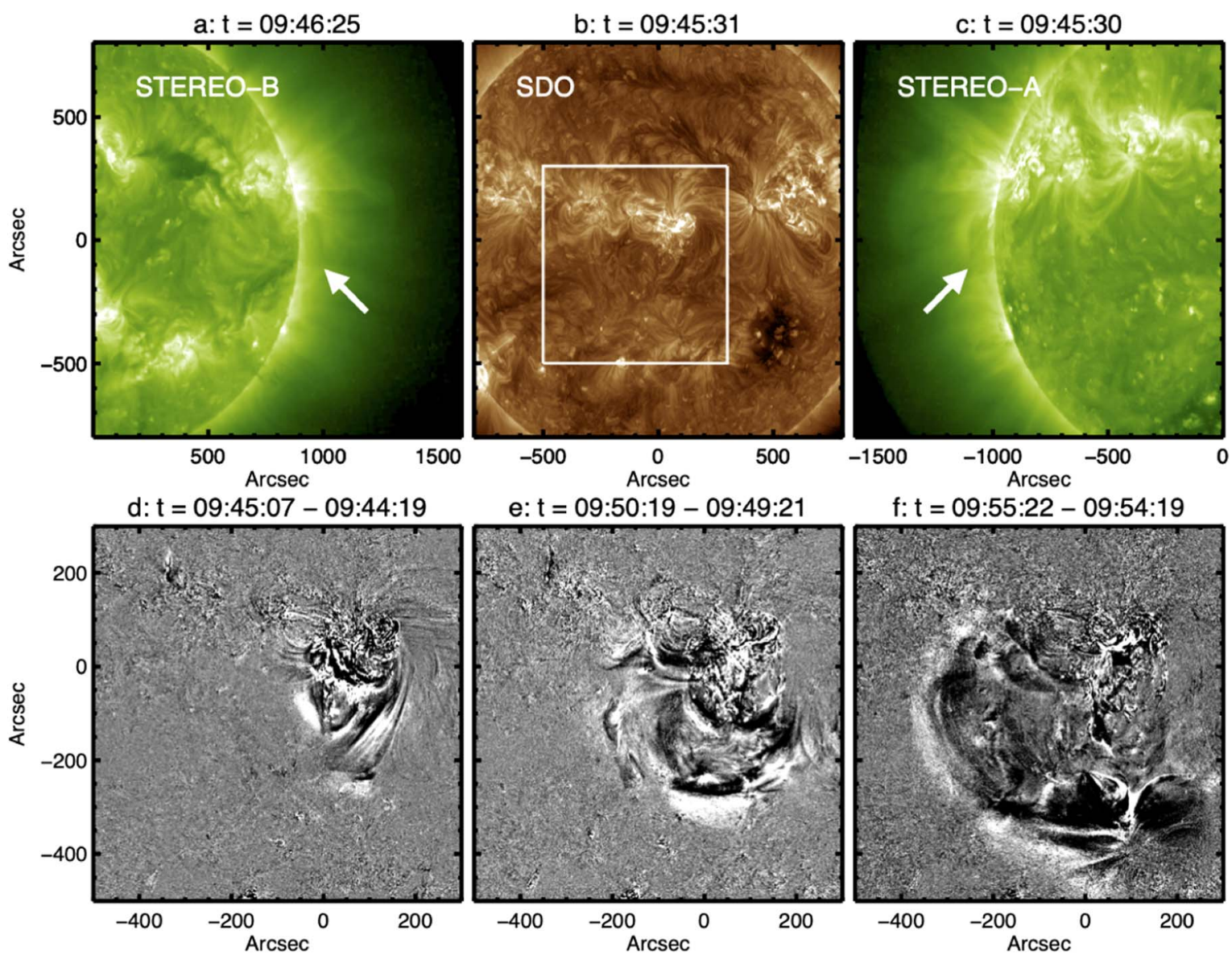

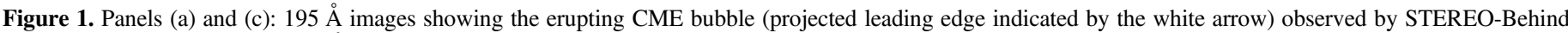

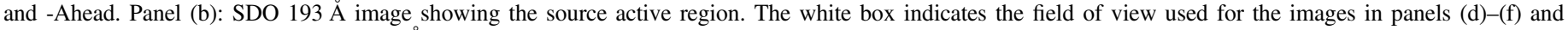

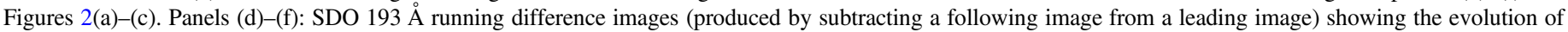
the global shock front.

resolved type III radio emission. The event and associated data sets are described in Section 2. The results are presented in Section 3, examining the evolution of the radio emission (Section 3.1), plasma signatures (Section 3.2), and the magnetic field (Section 3.3), before these diagnostics are discussed and some conclusions are drawn in Section 4.

\section{Observations and Data Analysis}

On 2011 October 1, an M1.2 solar flare erupted from NOAA Active Region (AR) 11305, near the center of the solar disk as seen from Earth. It was associated with an Earth-directed CME, and was well observed at EUV wavelengths by the Atmospheric Imaging Assembly (AIA; Lemen et al. 2012) onboard the SDO spacecraft near Earth and the Extreme Ultra-Violet Imagers (EUVI; Wuelser et al. 2004) onboard the Solar Terrestrial Relations Observatory (STEREO; Kaiser et al. 2008) Ahead and Behind spacecraft, which were $104^{\circ} .3$ and $97^{\circ} .5$ ahead of and behind the Earth, respectively, on its orbit around the Sun. The eruption was also associated with type II and III radio emission observed by the Nançay RadioHeliograph (NRH; Kerdraon \& Delouis 1997) and the Nançay Decameter Array (NDA; Lecacheux 2000). As it erupted, the CME drove a global shock wave which propagated primarily toward the southeast of the solar disk from the source active region.
The event was well observed by the STEREO and SDO spacecraft, with the data in each case reduced and processed using the standard SolarSoftWare routines (Freeland \& Handy 1998). Figure 1 shows the erupting CME as observed by the two STEREO spacecraft (panels (a) and (c)), with panel (b) showing the source active region as seen by the SDO spacecraft near Earth. The global wave can be identified as the white feature propagating away from the source active region in Figures 1(d)-(f), which highlight the feature using difference images (produced by subtracting an image from another taken at a later time). The associated radio emission is shown in Figure 2. Panels (a)-(c) in Figure 2 show the location and frequency of the radio emission at $50 \%$ of the peak intensity, while panels (d) and (e) show the radio spectra observed by NRH and NDA, respectively. The three vertical dashed lines in panel (e) correspond to the times of the images shown in panels (a)-(c). Panels (f) and (g) show a zoom-in on the NRH observations of the radio bursts (as indicated in panel (d)) offset from the active region identified in panels (a) and (b) without any interpolation in frequency.

The evolution of the global EUV wave was tracked on-disk using an intensity stack plot approach (see Long et al. 2019) in the $211 \AA$ passband observed by SDO/AIA (as this passband has been shown to be optimal for identifying and tracking global EUV waves, e.g., Long et al. 2014). A series of intensity stack plots similar to that shown in Figure 3(b) were used to 

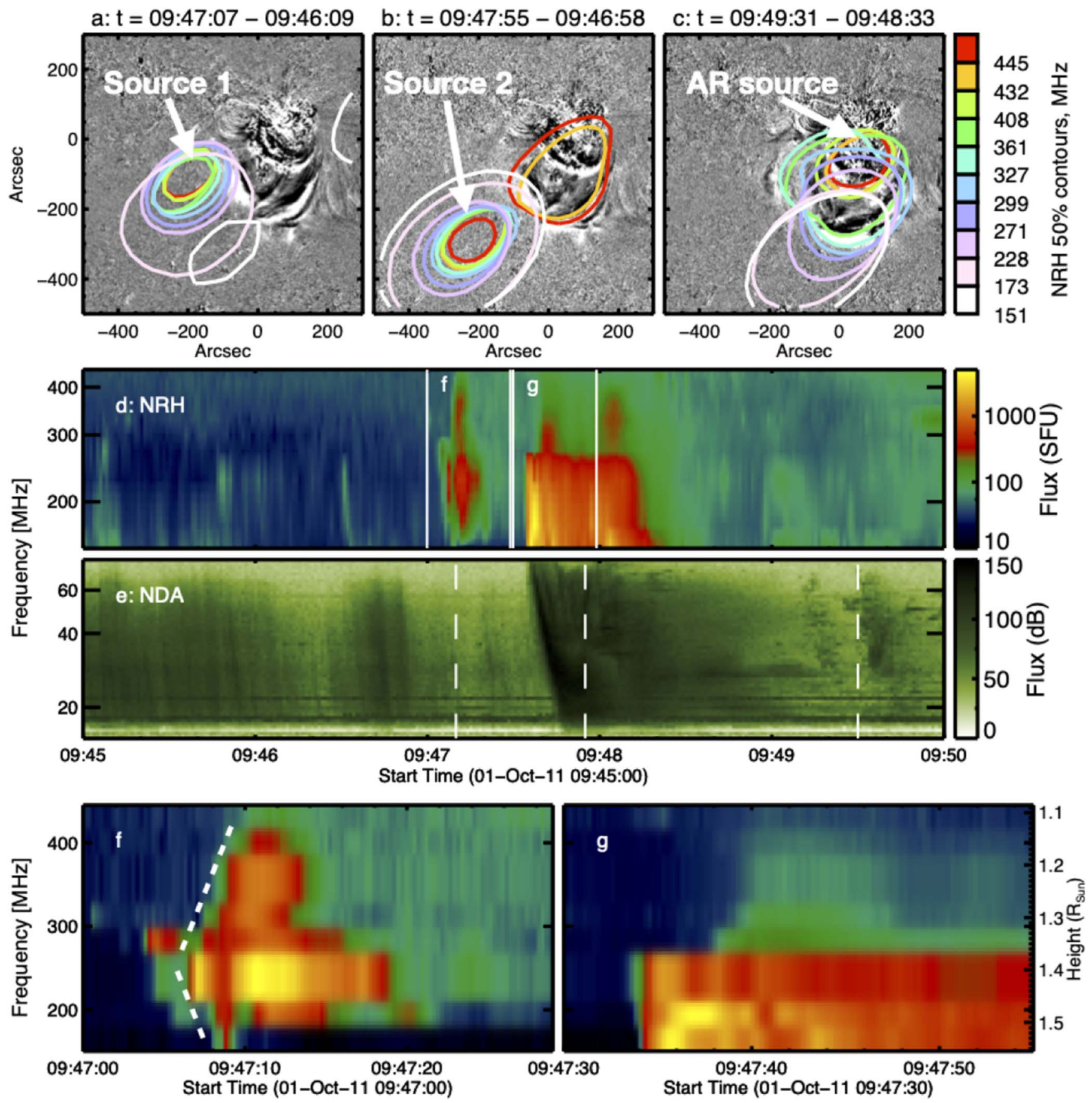

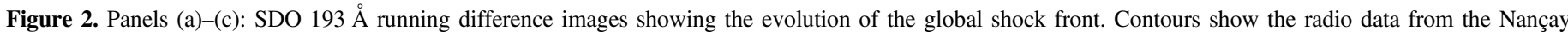

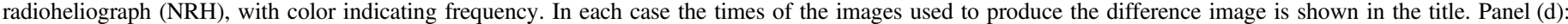

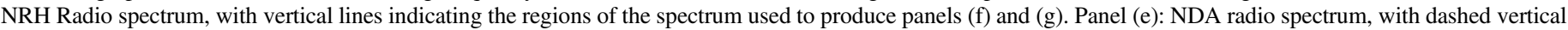

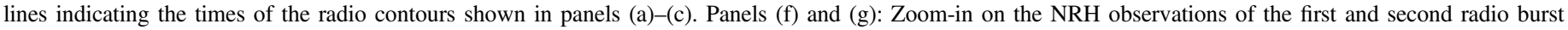
without any interpolation in frequency. Dashed white lines in panel (f) indicate the bifurcation of the radio signal as noted in the text.

track the temporal evolution of the global EUV wave, with the wave identified by eye 5 independent times, each using 100 points along a defined angle to minimize the associated uncertainty. All 500 points (as shown by the red dots in Figure 3(b)) were then used to determine the kinematics of the wave front along that angle using a quadratic fit (indicated by the blue line in Figure 3(b), with the fit parameters for this angle given in the panel legend). The process was repeated for each angle, enabling the spatial and temporal evolution of the global wave to be determined as shown in Figure 3(a), with the white line here corresponding to the intensity stack plot shown in panel (b).

The CME was observed by both STEREO spacecraft to erupt approximately in the plane of sky from the Earth-directed limb, enabling it to be tracked through the EUVI and Cor-1 fields of view. Figure 3(c) shows the STEREO-B EUVI and Cor-1 fields of view, with the white solid line indicating the angle used to derive the intensity stack plot shown in Figure 3(d). This angle (at $95^{\circ}$ clockwise from solar north) was chosen as it cut through the center of the plane-of-sky 

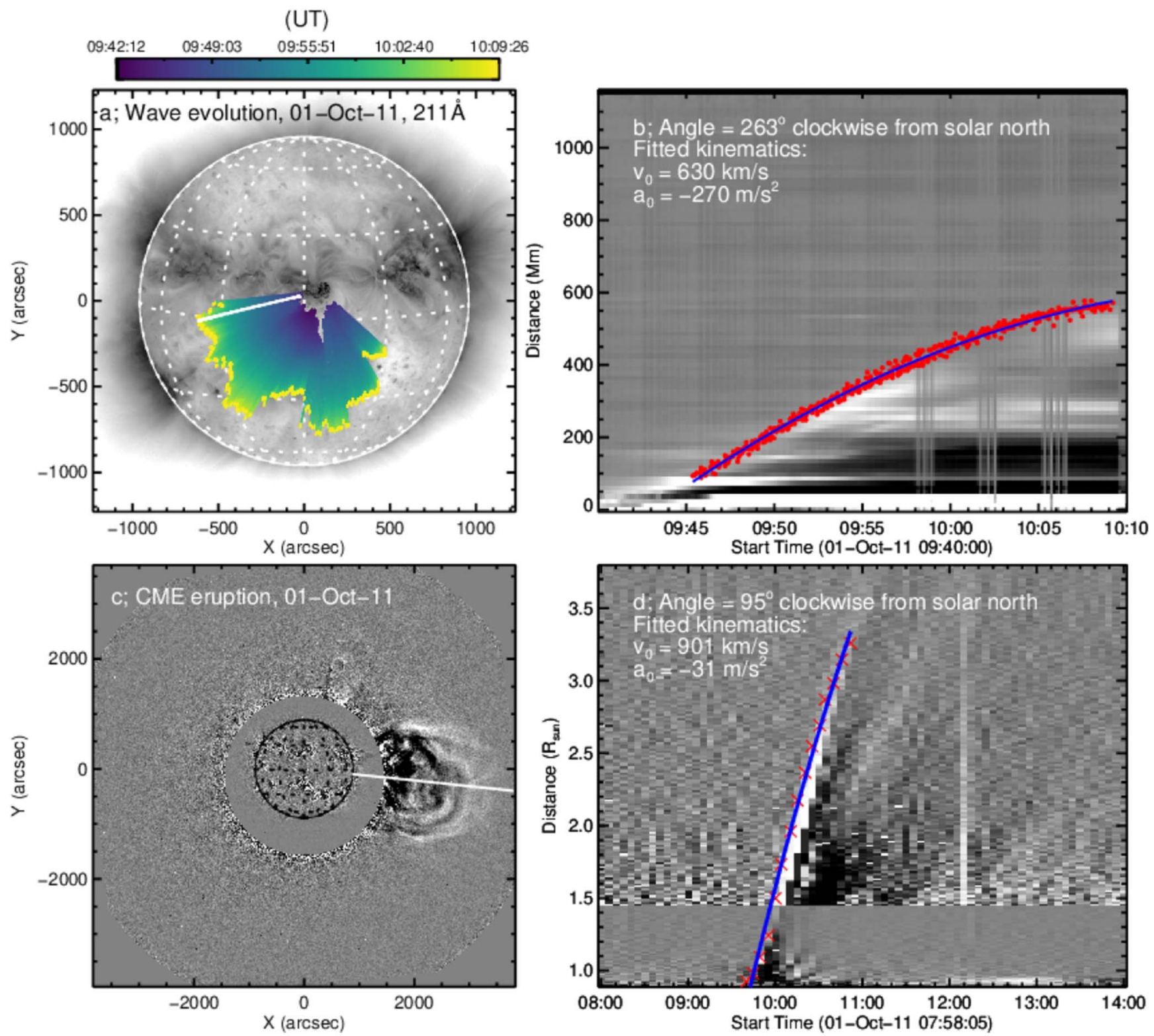

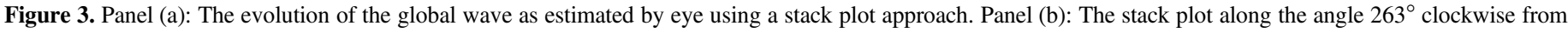

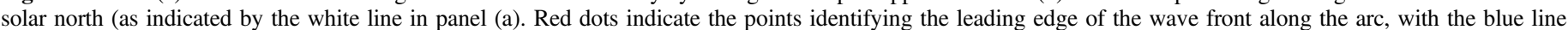

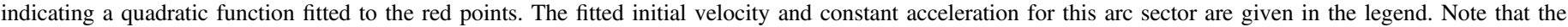

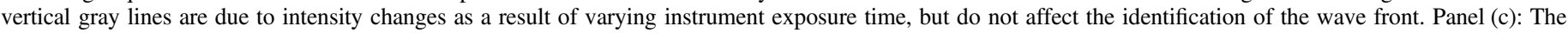

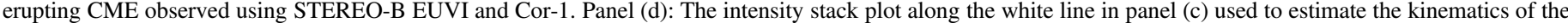

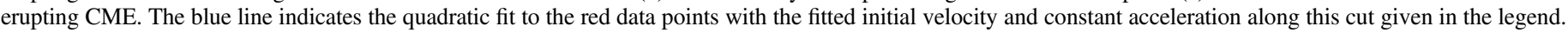

CME structure as it propagated through the field of view of both EUVI and Cor-1. The intensity along this line was then taken for each time step, with the bright edge of the CME identified manually and fitted using a quadratic function to derive its kinematics (following the stack plot approach described by O'Kane et al. 2019).

As shown in Figure 3(a), the global wave exhibited clearly anisotropic evolution, consistent with the initially southwarddirected eruption of the CME observed by both STEREO spacecraft and shown in Figure 1, and similar to the events previously studied by Long et al. (2019). However, it is worth noting that by the time the CME was observed by Cor- 1 onboard both STEREO spacecraft, it had become much more symmetric (as shown in Figure 3(c)), suggesting that by this time it was no longer constrained by the coronal magnetic field. The global wave was observed to propagate with an average initial velocity of $733 \mathrm{~km} \mathrm{~s}^{-1}$ and acceleration of $-427 \mathrm{~m} \mathrm{~s}^{-2}$, making it slower than previously studied global shock wave events (Long et al. 2015, 2017b), but still faster than global shocks observed using the previous generation of solar instrumentation (e.g., SOHO/EIT and STEREO/EUVI; see Thompson \& Myers 2009; Muhr et al. 2014). The CME was found to have a velocity of $901 \mathrm{~km} \mathrm{~s}^{-1}$ with an associated acceleration of $-31 \mathrm{~m} \mathrm{~s}^{-2}$ in the direction of Earth.

The NRH data at $0.25 \mathrm{~s}$ cadence was processed using the standard techniques and used for performing imaging spectroscopy between 450 and $150 \mathrm{MHz}$ to examine the type III radio emission associated with this global wave event (see Reid 2020, for a recent review). NDA spectroscopy data at $1 \mathrm{~s}$ cadence were used to examine the type III radio emission at frequencies between 80 and $14 \mathrm{MHz}$. A narrow type III radio burst can be observed in the radio 
spectrum shown in Figures 2(d) and 2(f) at t 09:47:10 UT, corresponding to source 1 identified in Figure 2(a). However, this type III burst is only observed by $\mathrm{NRH}$, indicating that it does not move to lower frequencies and hence higher altitudes. In contrast, an extended type III burst can be identified starting at $\mathrm{t} \sim$ 09:47:39 UT (Figure 2(g)), which is observed by both NRH and NDA. Figure 2(b) indicates that some of this emission comes from source 2, with a significant amount of emission also coming from the source active region (shown in Figure 2(c)). Although not seen in the timeframe shown here, a type II radio burst was also observed associated with this event starting at 09:55 UT, indicating the presence of a CME-driven shock in the outer corona.

\section{Results}

The radio emission associated with this event indicates the presence of a CME-driven shock (type II emission) and accelerated electrons (type III emission), both of which have previously been associated with global EUV waves (e.g., Harvey et al. 1974; Warmuth et al. 2004a, 2004b; Carley et al. 2013; Morosan et al. 2019). In this case, the type III emission was identified using imaging spectroscopy as coming from three distinct locations: the source active region and two sources ahead of the leading edge of the propagating wave front. These regions are identified by the white arrows in panels (a)-(c) of Figure 2; panels (a) and (b) show the sources associated with the propagating wave front (source 1 and 2) while panel(c) shows the active region source. Although sustained type III emission coming from the source active region can be explained by flare-driven electron acceleration, the origin of the short-lived emission coming from regions 1 and 2 identified in Figures 2(a) and (b) is less clear and requires further investigation.

\subsection{Radio Emission}

Panels (d) and (e) of Figure 2 show the dynamic spectra for the NRH and NDA instruments, respectively, in each case derived from the imaging spectroscopy shown in Figures 2(a)(c) using a field of view from $\left(-670^{\prime \prime},-670^{\prime \prime}\right)$ in the bottom left to $\left(287^{\prime \prime}, 287^{\prime \prime}\right)$ in the top right. Figures 2(f), (g) show zoomed-in sections of the NRH radio spectrum at the times of the radio bursts identified as occurring away from the source active region (in both cases with no interpolation across frequency). It is worth noting that whereas the zoom-in of source 1 (Figure 2(f)) shows a clear bifurcation of the radio emission (indicating electrons accelerated both toward and away from the Sun), the zoom-in of source 2 (Figure 2(g)) shows clear emission drifting to lower frequency but very weak emission drifting to higher frequency, indicating that the electrons were primarily propagating away from the Sun. Note that due to the very weak nature of the downward emission, it was not possible to estimate a drift rate for it.

We can identify properties of the particle acceleration region within source 1 (Figure 2(f)) using the type III radio emission. The density within the acceleration region can be estimated using the frequency at which the bidirectional type III bursts start. Assuming harmonic emission (due to the diffuse nature of the radio emission making it difficult for fundamental emission to escape the corona at higher frequencies, e.g., Reid 2020), this provides an electron density of $\sim 3.3 \times 10^{8} \mathrm{~cm}^{-3}$. The height of the acceleration region can then be estimated by assuming an electron density model and we use a $4 \times$ Saito model (Saito et al. 1977). As our radio source was on-disk, we could not use the source centroids to estimate the density model and so we chose the $4 \times$ multiplier to match the frequency of the bidirectional electron beams with the expected height range of the global EUV shock ( $70-100 \mathrm{Mm}$ above the photosphere; see Patsourakos \& Vourlidas 2009; Kienreich et al. 2009). While this assumption introduces a source of error, active regions typically have higher coronal densities than the quiet Sun which the standard Saito model represents. Assuming the $4 \times$ Saito model, the altitude at which the bifurcation occurs and hence where the particles are accelerated, corresponds here to $\sim 87 \mathrm{Mm}$ above the photosphere for source 1 . The relatively small frequency range where the type III emission starts indicates that the acceleration region is likely small in size, on the order of megameters or less, as the beam must quickly become unstable to Langmuir waves which produce the radio emission (Reid et al. 2014).

The velocity of the accelerated electron beams can be estimated using the assumed density model. A linear fit was made to the times of peak flux as a function of altitude, corresponding to the different frequencies observed by the $\mathrm{NRH}$. The $0.25 \mathrm{~s}$ time resolution of the NRH necessitated that the times of peak flux be estimated by fitting the light curves using a non-symmetric Gaussian function. The resultant velocities of the associated electron beams were $69 \mathrm{Mm} \mathrm{s}^{-1}$ and $82 \mathrm{Mm} \mathrm{s}^{-1}$ for the beams moving away from and toward the Sun, respectively. This equates to energies of $\sim 20 \mathrm{keV}$ and $\sim 28 \mathrm{keV}$, respectively. However, the electron beams consist of electrons with a distribution of velocities, traveling in a beamplasma structure due to wave-particle interactions (e.g., Reid \& Kontar 2018). If we assume that the maximum electron velocity within the beam is $1.5 x$ the derived velocity from the type III emission, the maximum energies of the electrons within the beams moving away from and toward the Sun increase to $44 \mathrm{keV}$ and $63 \mathrm{keV}$, respectively. It must also be noted that multiple crossings of the shock front would be required to achieve these energies (see Ball \& Melrose 2001).

For source 2 (Figure 2(g)), the downward emission is much weaker, with the upward emission originating at $\sim 230-270 \mathrm{MHz}$ (corresponding to a density of $2 \times 10^{8} \mathrm{~cm}^{-3}$ assuming harmonic emission). Although it was not possible to estimate a beam velocity for the upward propagating beam due to a lack of data points in the NRH observations, clear type III emission corresponding to source 2 is also observed in the NDA dynamic spectrum (Figure 2(e)). The $1 \mathrm{~s}$ time resolution of the NDA necessitated fitting the peak intensity as a function of frequency for each time bin using an asymmetric Gaussian so that the beam velocity could be found. A velocity of $79 \mathrm{Mm} \mathrm{s}^{-1}$ was found, similar to that of source 1 and with comparable energy. As for source 1, it was also possible to estimate the height of the particle acceleration for source 2 , corresponding to $\sim 137-185 \mathrm{Mm}$ above the photosphere for emission originating between 230 and $270 \mathrm{MHz}$.

\subsection{Plasma Diagnostics}

Next, the evolution of the coronal plasma associated with the passage of the global EUV shock front was further examined to identify variations in temperature and density. As discussed by Long et al. (2019), the spatial, temporal, and spectral resolution provided by SDO/AIA has enabled the development of a series of techniques designed to quantify the differential emission measure (DEM) of EUV plasma in the low solar corona (see, e.g., the work by Hannah \& Kontar 2013; Plowman et al. 2013; 

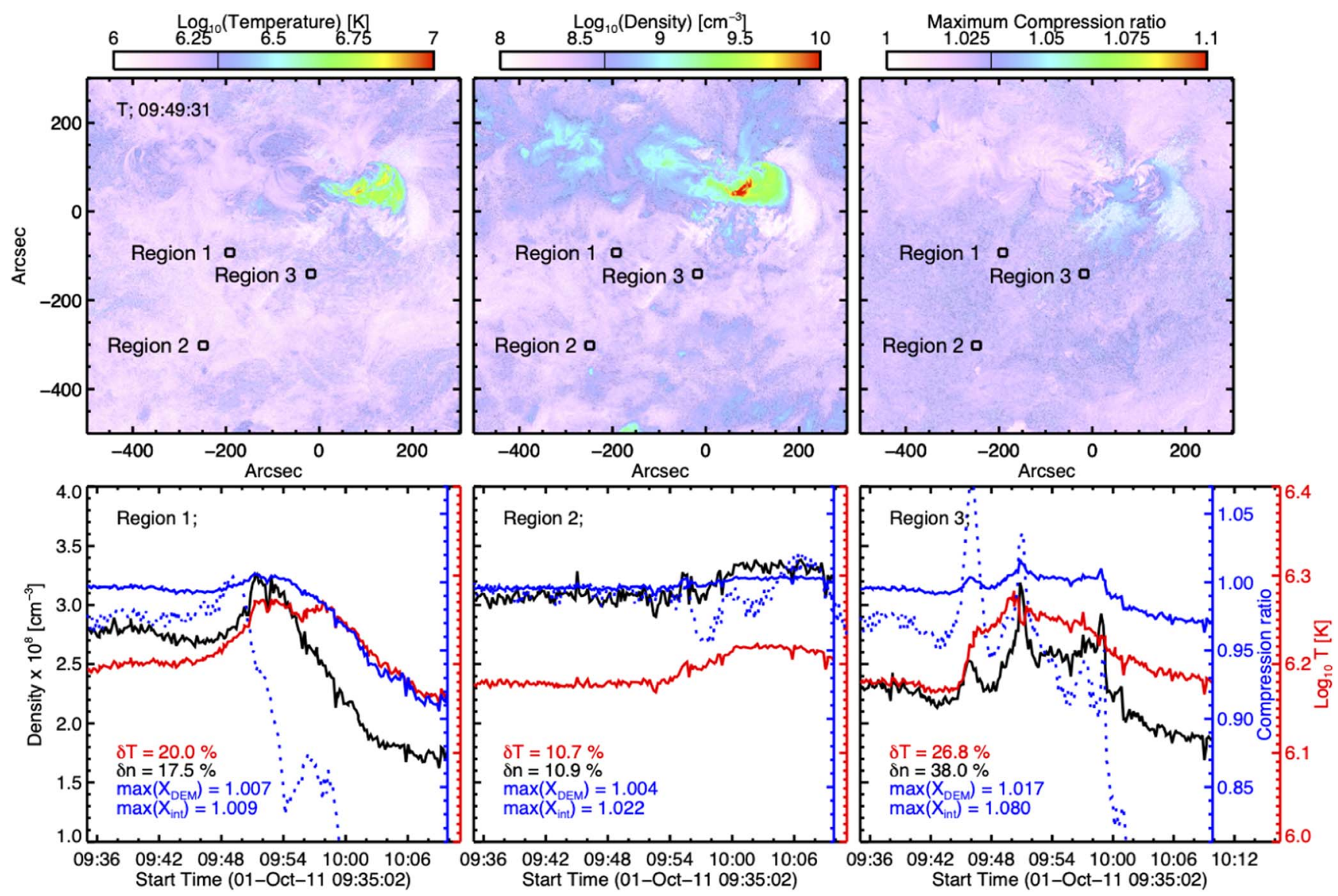

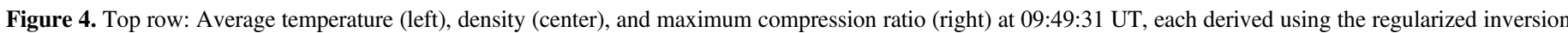

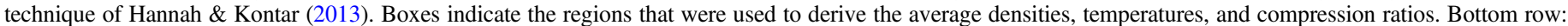

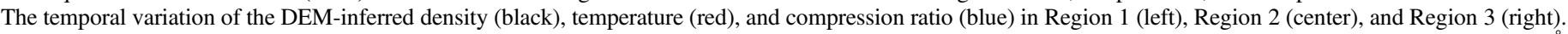

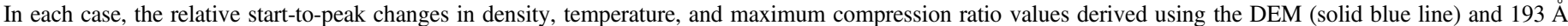
intensity (dashed blue line) approaches are indicated in the legend.

Cheung et al. 2015; Morgan \& Pickering 2019, for a number of different techniques designed to compute DEMs using observations from SDO/AIA). The DEM $\phi(T)$ is defined as,

$$
\phi(T)=n_{e}^{2}(T) \frac{d h}{d T},
$$

where $n_{e}$ is the density. Each of these techniques offers a different approach to solving this ill-posed problem, providing an opportunity to derive the DEM. This can then be used to derive the DEM-weighted density and temperature using the approach of Vanninathan et al. (2015) and Long et al. (2019). As noted by Cheng et al. (2012), the DEM-weighted density is defined as,

$$
n_{e}=\sqrt{\frac{\int \phi(T) d T}{h}},
$$

where $h$ is the plasma scale height. Similarly, the DEMweighted temperature is defined as,

$$
T=\sqrt{\frac{\int \phi(T) T d T}{\int \phi(T) d t}} .
$$

To quantify the role of the global wave in the origin of the type III radio emission labeled as sources 1 and 2 in Figure 2, the DEM of the plasma observed by SDO/AIA was estimated using the regularized inversion technique of Hannah \& Kontar (2013). This technique combines the 6 coronal EUV passbands observed by SDO/AIA $(94,131,171,193,211,335 \AA)$, enabling an estimate to be made of the emission measure of the region indicated by the white box in Figure 1(b). The DEMaveraged temperature of this region was estimated using Equation 3, and is shown in Figure 4(a) at 09:49:31 UT. The DEM-weighted density was estimated using Equation 2 with a scale height of $90 \mathrm{Mm}$ (see Patsourakos \& Vourlidas 2009; Vanninathan et al. 2015; Long et al. 2019), and is shown in Figure 4(b) at 09:49:31 UT. In both panels (a) and (b) of Figure 4, the temperature and density behave as expected, showing increased values in the core of the source active region, with lower values in the quiet Sun where the wave was observed to propagate.

Three regions of interest were identified to examine the temporal variation of the temperature and density; regions 1 (corresponding to radio source 1), 2 (corresponding to radio source 2), and 3 (representing the background quiet Sun). The variation in temperature (density) with time in each region is shown in red (black) in the lower row of Figure 4, which shows the variation in regions 1,2 , and 3 from left to right. Region 1, corresponding to radio source 1, exhibits increases of $\sim 20 \%$ and $17.5 \%$ above the pre-event temperature and density values, respectively, while region 2 which is located further from the source active region, exhibits a much smaller increase of $\sim 11 \%$ 
above the pre-event level in both temperature and density. Region 3, which is closer to the source active region than both regions 1 and 2 but does not have a corresponding radio source, exhibits higher increases in both temperature $(\sim 27 \%)$ and density $(\sim 38 \%)$. However, the density variation in region 3 is bursty and highly variable while the temperature is comparably smooth, with observations from the $304 \AA$ passband indicating that this bursty behavior is due to filamentary material associated with the core of the CME passing through the field of view.

The variation in density and temperature above the pre-event values observed in regions 1,2 , and 3 is proportional to their distance from the source active region, consistent with a radially expanding shock front (see Downs et al. 2021). However, the variation in temperature observed here is much larger than that observed for other, comparable global shock wave events (see Vanninathan et al. 2015; Long et al. 2019), suggesting a significant role for non-adiabatic processes. In particular, for both regions 1 and 3 (bottom left and right panels of Figure 4), the relative temperature and density increases are comparable, with sudden density increases at the front of the wave followed by a drop below the pre-event value while the temperature remains enhanced for a longer duration than the density before dropping back to its pre-event value. This suggests extended heating associated with the passage of the global wave front in each location close to the origin. In contrast, region 2 exhibits gradual and comparable increases in both density and temperature, with the return to the pre-event value occurring after the time-range examined here.

The DEM can also be used to estimate the density compression ratio and hence strength of the global shock wave. Although this is typically estimated using radio data by examining the difference in frequency between the fundamental and harmonic frequencies of the shock, the type II emission observed here corresponds to the leading edge of the CME-driven shock rather than the laterally driven global wave shock, and could therefore provide an inaccurate estimate of the shock strength. Instead, the density compression ratio can be estimated by examining the change in EUV intensity and/or DEM (see Kozarev et al. 2011; Ma et al. 2011; Muhr et al. 2011; Zhukov 2011; Frassati et al. 2019; Long et al. 2019; Frassati et al. 2020). The EUV density compression ratio $(X)$ was estimated here using both the intensity ratio approach of Zhukov (2011) and the DEM ratio approach of Frassati et al. (2019).

As described by Frassati et al. (2019), the density compression ratio can be estimated by examining the variation between pre- and post-event EM using the equation,

$$
X=\sqrt{\frac{\mathrm{EM}_{U}-\mathrm{EM}_{D}}{P_{U}}+1},
$$

where $\mathrm{EM}_{U}$ and $\mathrm{EM}_{D}$ are the upstream (i.e., ahead of the shock) and downstream (i.e., behind the shock) emission measure, respectively, and $P_{U}$ is the contribution to the preevent emission measure from coronal plasma compressed by the passage of the global wave (i.e., the plasma located between the points $L_{1}$ and $L_{2}$ along the line of sight). $P_{U}$ is defined as,

$$
\left.P_{U}=L<n_{e, U}^{2}>_{\text {LOS }} \text { (with } L=L_{2}-L_{1}\right) .
$$

Alternatively, the density compression ratio can be estimated using the approach of Zhukov (2011). Here, the density compression ratio can be related to the intensity ratio of the $193 \AA$ passband via the approximation,

$$
\frac{n}{n_{0}}=\sqrt{\frac{I}{I_{0}}},
$$

where $I_{0}$ and $n_{0}$ are the intensity and density, respectively, prior to the passage of the global wave. This approach only considers plasma in the temperature range of the $193 \AA$ passband $(T \sim 1$ $\mathrm{MK})$, and as a result is much more sensitive to the convolution of the timescale for ionization of the plasma with the timescale for increased plasma emission due to the passage of the shock (as discussed by Ma et al. 2011).

The variation in density compression ratio in each of the three regions estimated using both approaches is shown in the bottom row of Figure 4. Here, the solid blue line shows the density compression ratio estimated using the EM technique of Frassati et al. (2019), with the top right panel showing the maximum compression ratio across the whole field of view estimated using this technique. The dotted blue line in the bottom row of panels of Figure 4 shows the density compression ratio estimated using the $193 \AA$ intensity approach of Zhukov (2011). It is clear that while both compression ratio techniques provide similar results, there are distinct differences between them, with the compression ratio derived using the $193 \AA$ intensity approach exhibiting much more variability than the EM approach.

The evolution in density, temperature, and compression ratio shown in Figure 4 indicate that the global wave was freely propagating and very weakly shocked at the time and location of the observed type III radio emission. The estimated variation in density provides an excellent match to the radio emission, with the estimated height of the global wave and the starting frequency of the radio emission broadly consistent. The highly specific origins of the type III emission suggests that the shock wave may have locally created the conditions to accelerate the electrons that produced the observed emission. By combining the wave speed with the estimated density compression ratios in these locations, it is possible to infer the Mach number of the observed global wave.

Using the approach described in Section 2, the speed of the wave was determined at regions 1,2 , and 3 as identified in Figure 4. The global wave was found to have a velocity of $v=\sim 574 \mathrm{~km} \mathrm{~s}^{-1}$ at region 1 (corresponding to temperature and density increases of $\sim 20 \%$ and $17.5 \%$, respectively), a velocity of $v=\sim 410 \mathrm{~km} \mathrm{~s}^{-1}$ at region 2 (corresponding to temperature and density increases of $\sim 11 \%$ ) and a velocity of $v=\sim 408 \mathrm{~km} \mathrm{~s}^{-1}$ at region 3 (corresponding to temperature and density increases of $\sim 27 \%$ and $\sim 38 \%$, respectively). These observations are consistent with a shock wave propagating freely through the low solar corona.

The local sound speed $\left(c_{s}=\left(\gamma k_{B} T / m\right)^{1 / 2}\right)$ and thus hydrodynamic Mach number $\left(M=v_{\mathrm{sh}} / c_{s}\right)$ of the shock were estimated in each region of interest using the temperature derived above, and are listed in Table 1. It is clear that the wave was weakly shocked with a hydrodynamic Mach number of 1.4-1.9 depending on location.

The Alfvén Mach number $\left(M_{A}=v_{\mathrm{sh}} / v_{A}\right)$ and hence Alfvén speed $v_{A}$ can be estimated from the density compression ratio $(X)$ using the approach of Vršnak et al. (2002) via the 
Table 1

Plasma and Wave Properties

\begin{tabular}{lccc}
\hline \hline Property & Region 1 & Region 2 & Region 3 \\
\hline$v_{s h}\left(\mathrm{~km} \mathrm{~s}^{-1}\right)$ & 574.30 & 410.99 & 407.83 \\
$c_{s}\left(\mathrm{~km} \mathrm{~s}^{-1}\right)$ & 292.02 & 291.65 & 291.66 \\
$M=v_{\mathrm{sh}} / c_{s}$ & 1.967 & 1.409 & 1.398 \\
$v_{A}\left(\mathrm{~km} \mathrm{~s}^{-1}, \theta=0^{\circ}\right)$ & 572.42 & 407.61 & 407.24 \\
$\left.M_{A}=v_{\mathrm{sh}} / \nu_{A}, \theta=0^{\circ}\right)$ & 1.003 & 1.008 & 1.001 \\
$v_{A}\left(\mathrm{~km} \mathrm{~s}^{-1}, \theta=90^{\circ}\right)$ & 571.24 & 405.75 & 406.77 \\
$\left.M_{A}=v_{\mathrm{sh}} / v_{A}, \theta=90^{\circ}\right)$ & 1.005 & 1.013 & 1.003 \\
$B(\mathrm{G})$ & 3.5 & 2.5 & 2.5 \\
\hline
\end{tabular}

relationship,

$$
\begin{aligned}
& \left(M_{A}-X\right)^{2}\left[5 \beta X+2 M_{A}^{2} \cos ^{2} \theta(X-4)\right] \\
& \quad+M_{A}^{2} X \sin ^{2} \theta\left[(5+X) M_{A}^{2}+2 X(X-4)\right]=0,
\end{aligned}
$$

where $\beta$ is the ratio of plasma to magnetic pressure, and assuming an adiabatic index $\gamma=5 / 3$. In the case $\beta \rightarrow 0$, this reduces to,

$$
M_{A}=\sqrt{\frac{X(X+5)}{2(4-X)}},
$$

for a perpendicular shock $\left(\theta=90^{\circ}\right)$, and,

$$
M_{A}=\sqrt{X},
$$

for a parallel shock $\left(\theta=0^{\circ}\right)$. The resulting Alfvén speeds and Alfvén Mach numbers for $\theta=90^{\circ}$ and $\theta=0^{\circ}$ derived using the DEM density compression values given in Figure 4 are shown in Table 1. While there is very little difference between the derived Alfvén speed values for a perpendicular and parallel shock in each of the regions studied here, it is worth noting that perpendicular shocks are much more efficient at accelerating particles than parallel shocks.

The derived Alfvén velocity can also be used to estimate the magnetic field strength, $B$, of the three regions using the equation,

$$
B=v_{A} \sqrt{4 \pi n_{e} m_{i}} .
$$

The values of the Alfvén speed given in Table 1 give corresponding magnetic field strength values of $3.5 \mathrm{G}, 2.5 \mathrm{G}$, and $2.5 \mathrm{G}$ for regions 1,2 , and 3 , respectively. These estimates are consistent with previous estimates of the coronal magnetic field strength made using global EUV waves (see West et al. 2011; Long et al. 2013).

These measurements indicate that although the plasma diagnostics of the different regions of interest provide a useful insight into the ability of the studied global shock to produce the observed radio emission, they do not tell the whole story. In particular, the global shock studied here appears to be much too weak in the identified locations to produce the observed type III emission. However, the magnetic field through which the global shock propagated may provide some clues as to the origin of this emission.

\subsection{Magnetic Field Modeling}

To investigate the coronal magnetic field at the time of the eruption, a PFSS model was constructed using the Python pfsspy package (Yeates 2018; Stansby et al. 2020), with the
JSOC daily synoptic map of 30 September $2011^{3}$ providing the photospheric boundary condition and the source surface placed at 4 solar radii $\left(R_{s}\right)$. The pfsspy code was configured to internally remap the magnetogram using 360 nodes in longitude and 102 nodes in cosine-latitude, all uniformly spaced. In the radial direction, pfsspy employs a logarithmic grid, in this application using 120 nodes.

Figure 5 shows some of the field lines of the PFSS extrapolation, chosen using the field of view identified by the white box in Figure 1(b). As shown from the side in Figures 5(a), and as viewed from above in panel(b), the region of interest is entirely surmounted by the fan of a highaltitude null point (at approximately $0.28 R_{s}$ ), whose inner spine is rooted in the negative sunspot of the source active region while the outer spine connects back to the photosphere (at the northeast side). Such a strong topological constraint prevents any field line starting from within the area under the fan from connecting to interplanetary space.

This PFSS extrapolation provides some insight into the origin of the observed radio emission. As the origin of the radio emission is a function of coronal density, it is not possible to know the exact location of the radio emission in the 3D extrapolation without making some assumptions. First, we consider all the radio signals as coming from a single plane perpendicular to the line of sight at a height of around $1.2 R_{s}$ above the photosphere. Next, the helioprojective coordinates of the radio maps were used to align them to the PFSS extrapolation as seen from Earth. It should be noted that this is a largely arbitrary approach as it is physically incorrect to assume that the radio signals are coming from a planar surface (the height of which was estimated using average coronal plasma properties) and that such a planar surface is the same for all frequencies. However, these assumptions enable us to make a qualitative validation of the emission scenario that includes both magnetic field and radio emission. Figure 5(c) shows the result of such a comparison with selected field lines under the fan (yellow) and close to the null but external to the fan (dark green). The isocontours show the half-maximum NRH radio emission at 432 (orange), 228 (violet), and $151 \mathrm{MHz}$ (cyan) at 09:47:49 UT.

The emission corresponding to source 1 is associated with the field lines under the fan (yellow in Figure 5(c)) and is not connected to the magnetic null point, so the energetic particles are unable to escape into the heliosphere and instead are accelerated along closed magnetic loops. This matches the radio emission indicated by the first vertical dashed line in Figure 2(d), which was observed by NRH but not NDA (see the lack of a corresponding radio signature in Figure 2(e)). In contrast, the type III emission corresponding to source 2 and the active region source is connected to the magnetic null and hence open field lines (see dark green field lines in Figures 5(a), (c)). As the CME expands against the spine, it activates reconnection at the null. As a result, the energetic particles accelerated by the shock at source 2 and by ongoing reconnection in the active region can escape along these open magnetic field lines into the heliosphere. This matches the radio emission associated with the second vertical dashed line in Figure 2(e) which was observed by both NRH and NDA. The comparison between the CME bubble observed by STEREO and the field lines from the PFSS extrapolation (Figures 5(d),

\footnotetext{
hmi.mrdailysynframe_small_720s.20110930_120000_TAI.data.fits
} 

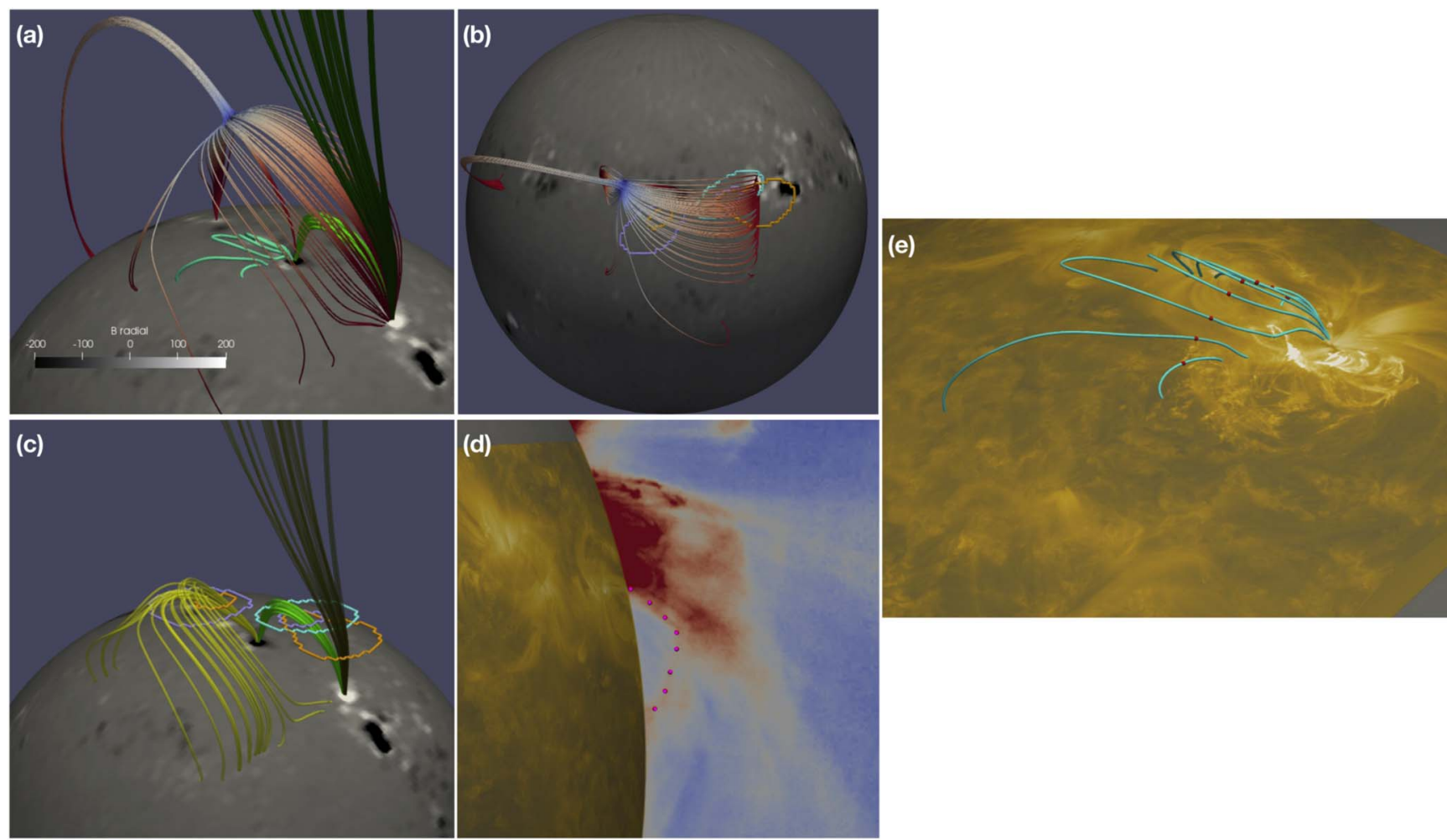

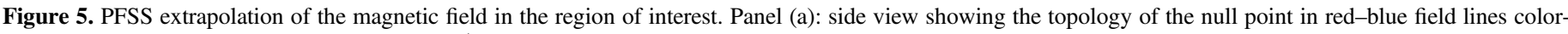

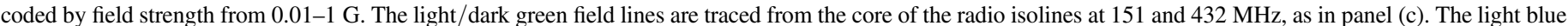

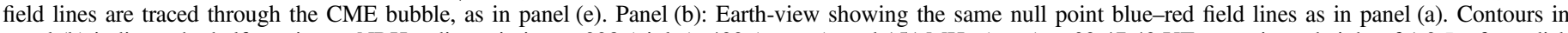

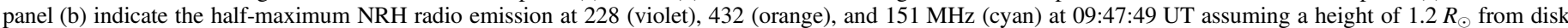

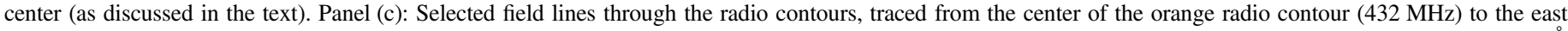

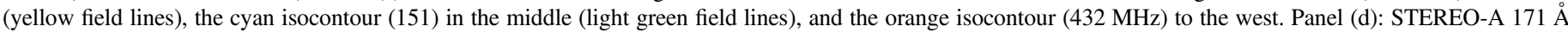

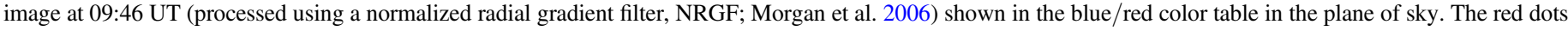

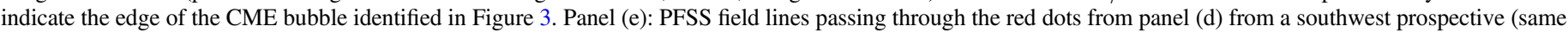

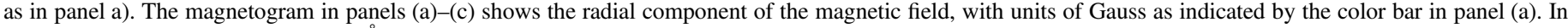
panels (d) and (e) the same AIA $171 \AA$ subdomain as outlined by the white square in Figure 2(b) is overlaid for reference.

(e)) show that the CME was still quite low when the type III emission was observed; consistent with the observations of the EUV wave. These side-on observations enable us to identify which field lines were impacted by the CME contemporaneously with the production of the type III radio signal. Figure 5(d) shows the size of the expanding bubble at 09:46 UT, the closest image available in time to the radio burst, identified by a series of red dots, as viewed from the STEREO perspective. The corresponding PFSS field lines originating from those dots are shown in Figure 5(e) as seen from a SE perspective, and in Figure 5(a) too for comparison with the fan structure.

Although the PFSS extrapolation does a good job of describing the static structure of the surrounding coronal magnetic field, it does not account for reconfiguration of the coronal magnetic field during an eruption. However, the combination of radio emission and PFSS extrapolation does provide unique insight into the configuration of the coronal magnetic field at the time of this eruption. In particular, the spine of the fan topology observed above the active region here enables particles accelerated by reconnection in the active region to escape into the heliosphere, resulting in the observed extended type III emission. The first type III burst (corresponding to the first burst observed ahead of the EUV wave) is produced by particles accelerated by the shock along closed magnetic field lines, while the second burst observed ahead of the EUV wave corresponds to the footpoints of magnetic loops connected to the null.

\section{Discussion and Conclusions}

As a well-observed, albeit weak, solar eruptive event with a CME, global shock wave, and associated radio emission, this event provides an excellent opportunity to study the acceleration of energetic particles by a weak shock. The global shock wave was tracked and analyzed using an intensity profile approach, and propagated anisotropically with a velocity of $\sim 400-500 \mathrm{~km} \mathrm{~s}^{-1}$ and a corresponding Alfvén Mach number of 1.001-1.013. In spite of this, it was able to accelerate electrons low down in the corona which produced distinct type III emission. However, the radio emission was highly localized, suggesting that only certain locations along the shock front achieved the required criteria for accelerating the energetic particles.

Global waves in the low solar corona have traditionally been interpreted as propagating through a radial coronal magnetic field and are often therefore interpreted as quasi-perpendicular shocks (see Carley et al. 2013). This quasiperpendicularity enables efficient acceleration of electrons via shock drift acceleration (Ball \& Melrose 2001), which involves adiabatic reflection of particles from the shock, with each reflection increasing the energy of the particle. For coronal shocks, the multiple reflection process has been explained using inhomogeneities in the shock 
front ("ripples"), with this quasiperiodic structure thought to explain the presence of herringbones in type II and III radio emission (e.g., Carley et al. 2013, 2015; Morosan et al. 2019). Here, source 1 appears to have a herringbone structure, but source 2 does not, suggesting a very limited deformation of the shock front assuming this interpretation.

The radio emission at both sources identified in Figures 2(a), (b) can be clearly identified to be ahead of the leading edge of the global EUV shock wave. This suggests that the radio emission observed here is due to a shock wave propagating ahead of the observed EUV front. In this case, the EUV observations provide a lower limit on the estimated kinematics of the shock front, as the shock must heat the plasma sufficiently to produce the observed perturbation (see Ma et al. 2011). We can then estimate the shock speed at $\sim 1.35$ times the observed EUV wave speed, assuming the shock starts at the same time and location as the EUV wave front and reaches the centroid of the observed radio source at the time of the observed radio emission.

To study this in more detail, both the plasma environment in the region through which the global shock wave evolved and the coronal magnetic field were examined using a regularized inversion DEM approach and a PFSS extrapolation, respectively. First, the plasma evolution was examined in the two regions identified as being the sources locations for the type III emission and a third region chosen as a background reference. All three regions were shown to exhibit increases in temperature and density associated with the passage of the global shock wave (see Figure 4). The variation in density and temperature measured in each region is proportional to the distance from the source active region, with the distinct, strong increases in both temperature and density observed in region 3 most likely due to blobs of plasma ejected by the active region passing through the field of view.

The DEM also enabled a detailed examination of the properties of the global EUV shock wave at the three regions of interest. In each location, the global EUV shock wave was found to be very weak, with a hydrodynamic Mach number ranging from 1.9 in region 1 to $\sim 1.4$ in regions 2 and 3 . However, the Alfvén Mach number was found to be much lower, ranging from $\sim 1.008$ (1.01) for an angle of $\theta=0^{\circ}$ $\left(\theta=90^{\circ}\right)$ between the magnetic field and shock normal in regions 1 and 2 to $\sim 1.001$ (1.003) for an angle of $\theta=0^{\circ}$ $\left(\theta=90^{\circ}\right)$ between the magnetic field and shock normal in region 3 . This indicates that while the global shock wave was stronger in the locations corresponding to the type III radio emission, it was still very weak, and most likely could not have produced the particle acceleration observed here.

The surrounding coronal magnetic field was then examined using a PFSS extrapolation. This investigation revealed a coronal null point above the source active region as shown in Figure 5(a), which suggests that the eruption occurred in a closed magnetic topology without direct access to a magnetic field opening into the heliosphere. However, the kinematic evolution of the erupting CME was found to be best fit by a quadratic function, kinematically consistent with an eruption evolving via a breakout model scenario (see Antiochos et al. 1999). The first type III emission corresponding to source 1 (see Figure 2(a)) was found to be due to particles accelerated both upward and downward along a closed magnetic loop, as evidenced by the bifurcated emission (Figure 2(f)) and lack of low-frequency emission associated with the burst. In contrast, the radio emission associated with source 2 (see Figure 2(b)) was due to particles accelerated upward away from the Sun along open magnetic field lines, as evidenced by the lack of a bifurcated signature in the NRH spectrum (Figure 2(g)) and the low-frequency emission observed by the NDA.

The event can be initially described as follows. Starting at 09:47 UT, we note a temporal correlation between the eruption and the observed radio signal, which includes type III bursts, indicating access to an open magnetic field. However, the source active region (AR 11305) is isolated from the open field, as it is fully surmounted by the fan of a (relatively) highaltitude null point, the inner spine of which is rooted in the active region. The earliest observation of the CME bubble by STEREO shows the bubble expanding but still far from the null point, with the only open field lines from the PFSS extrapolation rooted at the edge of the fan of the null, in the positive polarity of the neighboring AR 11306. Due to the proximity to the fan, these field lines spread out continuously from the spine of the null to the open field. We can propose the following scenario to explain these observations. The CME bubble expands in the SE direction, but also against the spine of the null rooted in the negative polarity of AR 11305. This activates reconnection at the null, impacting the dark green field lines shown in Figure 5(a), and eventually providing access to open field. This scenario is feasible as there are multiple ways for reconnection at the null to provide access to the dark green field lines in Figure 5(a). These include null deformation due to the expanding CME bubble (see Pontin et al. 2007; Galsgaard \& Pontin 2011), or slipping reconnection across the outer spine quasi-separatrix layer at the null (e.g., Masson et al. 2017). However, this hypothesis is limited by the potential nature of the PFSS extrapolation, which means that we cannot pinpoint a specific mechanism. As a result, we must limit our suggestion to a generic "activation" of the null point driven by the expanding CME bubble

The combination of ongoing reconnection in the source active region and the compression of the magnetic spine rooted in the active region by the erupting CME then resulted in continuous acceleration of energetic particles, producing the long-lasting type III emission identified as the AR source in Figure 2(c).

Therefore, rather than the bursty emission resulting from the repeated acceleration of electrons by the rippled front of the global shock wave as previously observed by Carley et al. (2013), Morosan et al. (2019), the radio emission observed here was produced by electrons accelerated by the interaction of the global shock with the surrounding coronal magnetic field. Although this scenario might be expected for every CME erupting into the surrounding coronal magnetic field, the very weak nature of the eruption and shock observed here enabled the properties of the global EUV wave and the associated spatially resolved type III radio emission to be disentangled from the main eruption and studied in detail for the first time. The ability of the Solar Orbiter and Parker Solar Probe to observe the solar corona at higher spatial and temporal resolution than currently possible and detect solar energetic particles closer to their source will enable a more thorough investigation and hence deeper understanding of this phenomenon.

We wish to thank the anonymous referee whose comments helped to improve the article. D.M.L. is grateful to the Science 
Technology and Facilities Council for the award of an Ernest Rutherford Fellowship (ST/R003246/1), and thanks ISSI (International Space Science Institute, Bern) for the hospitality provided to the team "Foreshocks Across the Heliosphere" led by H. Hietala and F. Plaschke, whose discussions helped to clarify the ideas described in this paper. G.V. acknowledges the support from the European Union's Horizon 2020 research and innovation program under grant agreement No. 824135 and of the STFC grant number ST/T000317/1. JO is supported by funding from the Science and Technology Facilities Council (STFC) studentship ST/R505171/1.

Facilities: SDO, NRH, STEREO, NDA.

Software: SolarSoftWare (Freeland \& Handy 1998); pfsspy (Yeates 2018; Stansby et al. 2020).

\section{ORCID iDs}

David M. Long (iD https://orcid.org/0000-0003-3137-0277 Hamish A. S. Reid (1) https://orcid.org/0000-0002-6287-3494 Gherardo Valori (iD https://orcid.org/0000-0001-7809-0067 Jennifer O'Kane (i) https://orcid.org/0000-0002-8806-5591

\section{References}

Antiochos, S. K., DeVore, C. R., \& Klimchuk, J. A. 1999, ApJ, 510, 485 Ball, L., \& Melrose, D. B. 2001, PASA, 18, 361

Carley, E. P., Long, D. M., Byrne, J. P., et al. 2013, NatPh, 9, 811

Carley, E. P., Reid, H., Vilmer, N., \& Gallagher, P. T. 2015, A\&A, 581, A100

Cheng, X., Zhang, J., Saar, S. H., \& Ding, M. D. 2012, ApJ, 761, 62

Cheung, M. C. M., Boerner, P., Schrijver, C. J., et al. 2015, ApJ, 807, 143

Downs, C., Warmuth, A., Long, D. M., et al. 2021, ApJ, 911, 118

Frassati, F., Mancuso, S., \& Bemporad, A. 2020, SoPh, 295, 124

Frassati, F., Susino, R., Mancuso, S., \& Bemporad, A. 2019, ApJ, 871, 212

Freeland, S. L., \& Handy, B. N. 1998, SoPh, 182, 497

Galsgaard, K., \& Pontin, D. I. 2011, A\&A, 534, A2

Hannah, I. G., \& Kontar, E. P. 2013, A\&A, 553, A10

Harvey, K. L., Martin, S. F., \& Riddle, A. C. 1974, SoPh, 36, 151

Kaiser, M. L., Kucera, T. A., Davila, J. M., et al. 2008, SSRv, 136, 5

Kerdraon, A., \& Delouis, J.-M. 1997, Coronal Physics from Radio and Space Observations; Proc. of the CESRA Workshop, ed. G. Trottet, (Berlin: Springer), 192

Kienreich, I. W., Temmer, M., \& Veronig, A. M. 2009, ApJL, 703, L118

Kozarev, K. A., Korreck, K. E., Lobzin, V. V., Weber, M. A., \& Schwadron, N. A. 2011, ApJL, 733, L25
Laitinen, T., \& Dalla, S. 2019, ApJ, 887, 222

Lecacheux, A. 2000, GMS, 119, 321

Lemen, J. R., Title, A. M., Akin, D. J., et al. 2012, SoPh, 275, 17

Long, D. M., Baker, D., Williams, D. R., et al. 2015, ApJ, 799, 224

Long, D. M., Bloomfield, D. S., Chen, P. F., et al. 2017b, SoPh, 292, 7

Long, D. M., Bloomfield, D. S., Gallagher, P. T., \& Pérez-Suárez, D. 2014, SoPh, 289, 3279

Long, D. M., Jenkins, J., \& Valori, G. 2019, ApJ, 882, 90

Long, D. M., Murphy, P., Graham, G., Carley, E. P., \& Pérez-Suárez, D. 2017a, SoPh, 292, 185

Long, D. M., Williams, D. R., Régnier, S., \& Harra, L. K. 2013, SoPh, 288, 567

Ma, S., Raymond, J. C., Golub, L., et al. 2011, ApJ, 738, 160

Masson, S., Pariat, É., Valori, G., et al. 2017, A\&A, 604, A76

Morgan, H., Habbal, S. R., \& Woo, R. 2006, SoPh, 236, 263

Morgan, H., \& Pickering, J. 2019, SoPh, 294, 135

Morosan, D. E., Carley, E. P., Hayes, L. A., et al. 2019, NatAs, 3, 452

Morosan, D. E., Palmerio, E., Lynch, B. J., \& Kilpua, E. K. J. 2020, A\&A, 633, A141

Muhr, N., Veronig, A. M., Kienreich, I. W., et al. 2014, SoPh, 289, 4563

Muhr, N., Veronig, A. M., Kienreich, I. W., Temmer, M., \& Vršnak, B. 2011, ApJ, 739, 89

Nitta, N. V., Schrijver, C. J., Title, A. M., \& Liu, W. 2013, ApJ, 776, 58

O'Kane, J., Green, L., Long, D. M., \& Reid, H. 2019, ApJ, 882, 85

Patsourakos, S., \& Vourlidas, A. 2009, ApJL, 700, L182

Pesnell, W. D., Thompson, B. J., \& Chamberlin, P. C. 2012, SoPh, 275, 3

Plowman, J., Kankelborg, C., \& Martens, P. 2013, ApJ, 771, 2

Pontin, D. I., Bhattacharjee, A., \& Galsgaard, K. 2007, PhPl, 14, 052109

Prise, A. J., Harra, L. K., Matthews, S. A., Long, D. M., \& Aylward, A. D. 2014, SoPh, 289, 1731

Reid, H. A. S. 2020, FrASS, 7, 56

Reid, H. A. S., \& Kontar, E. P. 2018, ApJ, 867, 158

Reid, H. A. S., Vilmer, N., \& Kontar, E. P. 2014, A\&A, 567, A85

Rouillard, A. P., Sheeley, N. R., Tylka, A., et al. 2012, ApJ, 752, 44

Saito, K., Poland, A. I., \& Munro, R. H. 1977, SoPh, 55, 121

Stansby, D., Yeates, A., \& Badman, S. 2020, JOSS, 5, 2732

Thompson, B. J., \& Myers, D. C. 2009, ApJS, 183, 225

Vanninathan, K., Veronig, A. M., Dissauer, K., et al. 2015, ApJ, 812, 173

Vršnak, B., Magdalenić, J., Aurass, H., \& Mann, G. 2002, A\&A, 396, 673

Warmuth, A. 2015, LRSP, 12, 3

Warmuth, A., Vršnak, B., Magdalenić, J., Hanslmeier, A., \& Otruba, W. 2004a, A\&A, 418, 1101

Warmuth, A., Vršnak, B., Magdalenić, J., Hanslmeier, A., \& Otruba, W. 2004b, A\&A, 418, 1117

West, M. J., Zhukov, A. N., Dolla, L., \& Rodriguez, L. 2011, ApJ, 730, 122

Wuelser, J.-P., Lemen, J. R., Tarbell, T. D., et al. 2004, Proc. SPIE, 5171, 111

Yeates, A. 2018, antyeates1983/pfss: First release of pfss code., v1.0, Zenodo doi: $10.5281 /$ zenodo. 1472183

Zhukov, A. N. 2011, JASTP, 73, 1096 